\title{
Labyrinthe
}

19 | 2004 (3)

Le Bel Aujourd'hui

\section{La théorie cartésienne du jugement}

\section{Élodie Cassan}

\section{OpenEdition}

\section{Journals}

Édition électronique

URL : http://journals.openedition.org/labyrinthe/251

DOI : $10.4000 /$ labyrinthe.251

ISSN : 1950-6031

Éditeur

Hermann

Édition imprimée

Date de publication : 15 décembre 2004

Pagination : 135-138

Référence électronique

Élodie Cassan, "La théorie cartésienne du jugement », Labyrinthe [En ligne], 19 | 2004 (3), mis en ligne le 19 juin 2008, consulté le 02 mai 2019. URL : http://journals.openedition.org/labyrinthe/251 ; DOI : $10.4000 /$ labyrinthe.251

Propriété intellectuelle 


\title{
LA THÉORIE CARTÉSIENNE DU JUGEMENT*
}

\author{
Élodie CASSAN \\ cassan@univ-paris12.fr
}

Les Méditations métaphysiques (1641) de Descartes proposent une double caractérisation psychologique et logique du jugement. Si l'acte de juger est imputé à la volonté, faculté d'affirmer ou de nier une chose, cette puissance n'évite l'erreur que pour autant qu'elle suit « la résolution de ne juger jamais d'aucune chose sans la concevoir clairement et distinctement ${ }^{1} »$. Des textes de Descartes consacrés au jugement, la Méditation quatrième est le seul à faire explicitement dépendre le jugement de la volonté. Est-ce à dire que la théorie cartésienne du jugement s'épuise dans ce seul passage? Des textes antérieurs aux Méditations traitent du jugement. Quel statut leur attribuer? Servent-ils seulement à fournir le cadre conceptuel d'une théorie du jugement marquée par l'unicité? Constituent-ils au contraire une preuve que cette théorie évolue tout au long de l'œuvre de Descartes? Prendre position sur cette question requiert non seulement d'identifier la place accordée par Descartes au concept de jugement dans son œuvre avant 1641, mais aussi de reconstruire les différents sens de ce concept au sein de cet ensemble ${ }^{2}$.

De façon générale, l'acte de juger intervient dans le contexte de la recherche de la vérité. Ainsi par exemple dans le Discours de la méthode (1637), où si « la puissance de bien juger, et distinguer le vrai d'avec le faux, qui est proprement ce que l'on nomme le bon sens ou

\footnotetext{
* Le présent article donne les principales orientations d'une thèse d'histoire de philosophie («La théorie cartésienne du jugement»), préparée sous la direction du professeur Frédéric de Buzon, à l'université Paris XII.

1. Méditations métaphysiques, Paris, Édition Adam et Tannery (= A. T.), tome IX-a, p. 49.

2. Ce travail est rendu légitime par l'idée que «It matters not at all whether Descartes' or Aristotle's or Kant's view are true or false for this use of history. What is important is that we understand what their view were, and that we understand how it is that smart people have regarded them as true ", Daniel Garber, Descartes embodied, Cambridge, Cambridge University Press, 2001, p. 24.
} 
la raison, est naturellement égale en tous les hommes [au demeurant] ce n'est pas assez d'avoir l'esprit bon, mais le principal est de l'appliquer bien³.» Dans ce texte, ce qui rend un jugement bon n'est pas analysé en termes moraux. En effet, bien juger ce n'est pas exercer suffisamment de discernement pour parvenir à l'emporter sur l'irrésolution, l'inconstance et l'instabilité, à la différence de ce que Charron établit dans De la sagesse ${ }^{4}$. Par ailleurs, la rectitude du jugement n'est pas comprise en termes juridiques. Alors que dans le dictionnaire de Nicot (Trésor de la langue française, 1606) de nombreuses entrées associent justice et jugement ${ }^{5}$, Descartes fait intervenir ce dernier dans un cadre épistémologique. Ainsi, un jugement est bon si, et seulement si, il procède de l'application ingénieuse de la pensée à un problème donné. Qu'est-ce à dire ?

Pour déterminer dans quelle mesure un jugement est bon et par là contribue au progrès de la connaissance, il convient de s'entendre au préalable sur ce que le concept de jugement signifie en lui-même. Mais à ce sujet, l'univocité n'est pas de mise. D'abord, selon les Règles pour la direction de l'esprit (composées avant 1629), le jugement compose les notions que nous avons des choses, les natures simples, appréhendées au préalable dans l'intuition. "Par exemple ; si je juge qu'une certaine figure n'est pas en mouvement, je dirai que la pensée que j'en ai se compose en quelque sorte de la figure et du repos ${ }^{6}$.» L'idée du jugement comme composition est reprise de la scolastique tardive. Ainsi, dans la Summa Philosophiae Quadripartita (1609), Eustache de saint Paul écrit: "Cum affirmamus:hominem esse animal, ipsum

\footnotetext{
3. René Descartes, Paris, A. T., tome VI, p. 1-2.

4. «L'homme est un sujet merveilleusement divers et ondoyant, sur lequel il est très malaisé d'y asseoir jugement assuré, jugement dis-je, universel et entier, à cause de la grande contrariété et dissonance des pièces de notre vie. La plupart de nos actions ne sont que saillies et bouttées poussées par quelques occasions : ce ne sont que pièces rapportées. L'irrésolution d'une part, puis l'inconstance et l'instabilité, est le plus commun et apparent vice de la nature humaine», Charron, De la sagesse, Livre I, chapitre 5. Texte consulté sur le site http://www.gallica.bnf.fr

5. On trouve par exemple: «Jugement donnant instruction à d'autres juges, en semblables matieres, ou autres ayans connexité et dependance de la premiere», "Comparoistre en jugement». Une très bonne édition électronique de ce texte est proposée sur le site: http://www.lib.uchicago.edu/efts/ARTFL/newhome/ref/

6. Règles pour la direction de l'esprit, traduction J. Brunschwig, Classiques Garnier, Éditions de F. Alquié, 1988, p.146. Pour le texte original, en latin, voir Regulae ad directionem ingenii, A. T., tome X, p. 420.
} 
Animal cum Homine componimus ${ }^{7}$.» Autrement dit, juger que l'homme est un animal consiste à composer les notions d'animal et d'homme. Ensuite, dans le Discours de la méthode, l'idée du jugement comme composition se voit concurrencée par une approche du jugement comme raison, capacité à s'exprimer en connaissance de cause. Tel est le sens qui se dégage de la critique de l'art de Lulle, qui permet de «parler, sans jugement ${ }^{8} »$. À ce point, il ne suffit donc pas d'énoncer formellement des jugements. Encore faut-il les produire en connaissance de cause.

Dès lors, en quoi le jugement est-il fondé à participer du progrès de la connaissance ? Pareille question se pose parce que la connaissance prend appui d'abord sur les idées; «l'idée est la chose même conçue, ou pensée, en tant qu'elle est objectivement dans l'entendement ${ }^{9} \gg$. En d'autres termes, avec Descartes le concept d'idée a pour lieu la science de l'homme et non celle de Dieu, à la différence de chez Thomas d'Aquin ${ }^{10}$. Si le concept d'idée est central, pourquoi le concept de jugement ne s'y voit-il pas réduit? À quels points de vue la théorie du jugement constitue-t-elle un complément de la théorie de l'idée? Par là, il apparaît très clairement que l'enjeu de la présente recherche est de contribuer à éclairer la conception cartésienne de la science.

À ce titre, il convient de se demander comment, une fois formé dans un cadre épistémologique, le jugement en vient à recouvrir différents champs d'application. D'une part, à propos des connaissances obtenues en physique, les Principes de la philosophie (1644) établissent que l'«on ne laissera pas d'avoir pour le moins autant de raison de juger qu'elles sont les vraies causes de tout ce que j'en ai déduit, qu'on en a de croire qu'on a trouvé le vrai sens d'un chiffre, lorsqu'on le voit suivre de la signification qu'on a donnée par conjecture à chaque lettre $^{11} \gg$. Comment le concept de jugement peut-il servir à décrire le type de certitude obtenu en physique?

7. Summa Philosophiae Quadripartita de rebus dialecticis, moralibus, physicis et metaphysicis, Secunda pars Dialecticae, Praefatio. Texte consultable sur le site: http://gallica.bnf.fr/

8. Discours de la méthode, A. T., tome VI, p. 17.

9. Réponses aux Premières Objections, A. T., tome IX-a, p. 81.

10. Le caractère déterminant de ce changement a été mis en évidence notamment lors du séminaire de maîtrise du professeur M. Fichant, portant sur «La notion d'idée de Descartes à Leibniz», et qui s'est tenu à la Sorbonne au premier semestre 2003-2004.

11. Principes de la philosophie, A. T., tome IX-b, p. 323-324. 
Par ailleurs, si le jugement relève avant tout du domaine théorique, il intervient également en morale. Il est ainsi question dans les Passions de l'âme (1649) de «jugements fermes et déterminés touchant la connaissance du bien et du mal ${ }^{12} \gg$. Comment théorique et moral sontils articulés? Le jugement pratique peut-il être un cas particulier du jugement de connaissance?

$\mathrm{Au}$ bout du compte, cette recherche permet d'accéder aux différentes branches de la pensée de Descartes, et de les relier de façon nouvelle. Cette diversité d'entrées dans le corpus est à l'image de la multiplicité des impacts que le concept de jugement y laisse. Par là, elle laisse à penser que tout n'est pas dit de ce dernier dans les Méditations métaphysiques.

12. Passions de l'âme, art. 48, A. T., tome XI, p. 367. 